NISSUNA UMANA INVESTIGAZIONE SI PUO DIMANDARE VERA SCIENZIA S'ESSA NON PASSA PER LE MATEMATICHE DIMOSTRAZIONI LEONARDO DA VINCI

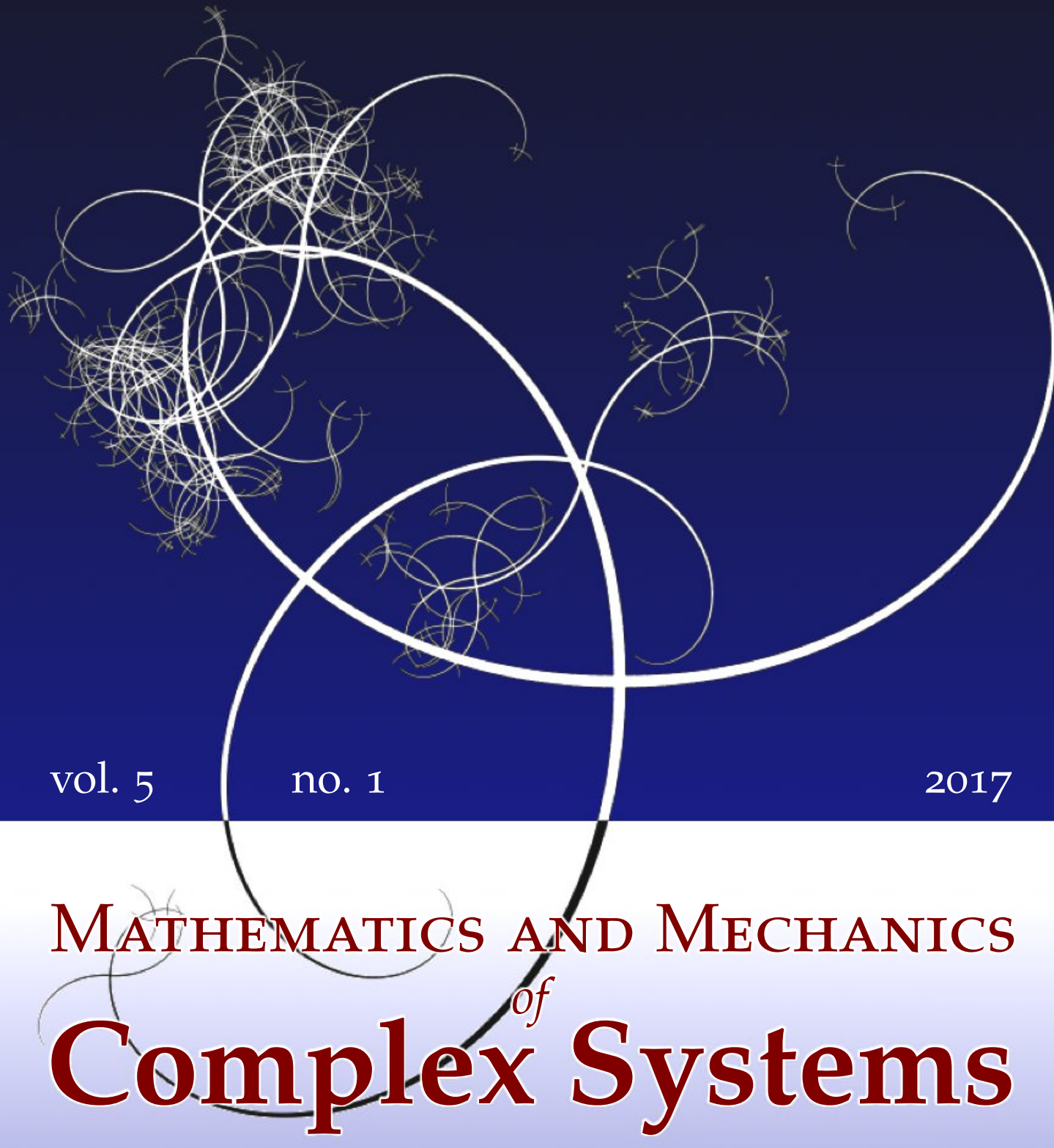

HARM ASKES AND INNA M. GitMAN

REDUCIBLE AND IRREDUCIBLE FORMS OF STABILISED GRADIENT ELASTICITY IN DYNAMICS 


\title{
REDUCIBLE AND IRREDUCIBLE FORMS OF STABILISED GRADIENT ELASTICITY IN DYNAMICS
}

\author{
HARM ASKES AND INNA M. GitMAN
}

\begin{abstract}
The continualisation of discrete particle models has been a popular tool to formulate higher-order gradient elasticity models. However, a straightforward continualisation leads to unstable continuum models. Padé approximations can be used to stabilise the model, but the resulting formulation depends on the particular equation that is transformed with the Padé approximation. In this contribution, we study two different stabilised gradient elasticity models; one is an irreducible form with displacement degrees of freedom only, and the other is a reducible form where the primary unknowns are not only displacements but also the Cauchy stresses - this turns out to be Eringen's theory of gradient elasticity. Although they are derived from the same discrete model, there are significant differences in variationally consistent boundary conditions and resulting finite element implementations, with implications for the capability (or otherwise) to suppress crack tip singularities.
\end{abstract}

\section{Introduction}

Gradient elasticity is a methodology to enrich the continuum equations of elasticity with additional higher-order spatial (and occasionally temporal) derivatives of certain state variables. There are different versions of gradient elasticity, such as those equipped with strain gradients, stress gradients and acceleration gradients; see for instance [Askes and Aifantis 2011] for a recent (but by no means complete) review.

Certain formats of gradient elasticity bear a close relationship with discrete lattice models of materials with microstructure; indeed, it is often possible to derive gradient elasticity theories by continualising the response of a discrete model, for instance using Taylor series approximations [Chang and Gao 1995; Mühlhaus and Oka 1996; Suiker et al. 2001a; Suiker et al. 2001b; Ioannidou et al. 2001; Askes and Metrikine 2005]. However, such models often suffer from intrinsic deficiencies, such as loss of stability in dynamics and loss of uniqueness in statics [Askes et al. 2002]. This can be amended by applying Padé approximations or similar

\section{Communicated by Francesco dell'Isola.}

MSC2010: 74-XX.

Keywords: gradient elasticity, mixed formulation, length scale, nonlocal elasticity. 


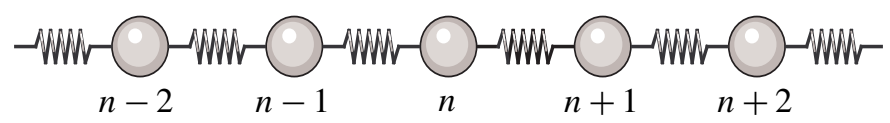

Figure 1. One-dimensional chain of masses connected by springs.

techniques, as has for instance been demonstrated in [Rosenau 1984; Rubin et al. 1995; Chen and Fish 2001; Andrianov 2002; Andrianov et al. 2003; Charlotte and Truskinovsky 2008]. Thus, stabilised gradient elasticity theories can be formulated that maintain their close link with discrete lattice models, thereby facilitating simple identification of the higher-order constitutive parameters (usually known as "intrinsic length scales" or "microstructural length scales").

In this paper, we compare two versions of stabilised gradient elasticity. Both can be derived from the response of a discrete lattice model, which is shown for the one-dimensional case. Variational formulations are presented for the multidimensional extensions. Throughout, a distinction is made between the so-called irreducible form where the only unknowns are the displacements and the reducible form where the unknowns are the displacements as well as the Cauchy stresses. The difference between these two forms has important consequences for the variationally consistent boundary conditions and finite element implementations. A numerical example will show the ability (or otherwise) of the two formulations to suppress singularities - this has historically been an important motivation for using gradient elasticity theories, and certain formats have been demonstrated to remove singularities even under restrictive conditions such as anisotropic material behaviour and bimaterial interface cracks [Kwong and Gitman 2012]. We also discuss the relation of the reducible form with Eringen's [1983] differential theory of nonlocal elasticity.

\section{Continualisation of the response of a discrete chain}

To illustrate the concepts of continualisation (this section) and stabilisation via Padé approximations (Section 3), the one-dimensional chain of particles and springs in Figure 1 is studied. All particles have mass $M$, and all springs have stiffness $K$. Furthermore, the interparticle distance is denoted by $d$. The equation of motion of particle $n$ thus reads

$$
M \ddot{u}_{n}=K\left(u_{n+1}-2 u_{n}+u_{n-1}\right)
$$

where $u_{i}$ is the displacement of particle $i$. A continuum approximation is obtained by replacing $u_{n}$ with $u(x)$ and $u_{n \pm 1}$ with $u(x \pm d)$. Taylor series expansions are applied according to

$$
u(x \pm d)=u(x) \pm d \frac{\partial u}{\partial x}+\frac{1}{2} d^{2} \frac{\partial^{2} u}{\partial x^{2}} \pm \frac{1}{6} d^{3} \frac{\partial^{3} u}{\partial x^{3}}+\frac{1}{24} d^{4} \frac{\partial^{4} u}{\partial x^{4}} \pm \cdots
$$


so that (1) can be rewritten as

$$
\rho \ddot{u}=E\left(\frac{\partial^{2} u}{\partial x^{2}}+\frac{1}{12} d^{2} \frac{\partial^{4} u}{\partial x^{4}}+\cdots\right)
$$

where the mass density $\rho=M / A d$ and the Young's modulus $E=K d / A$, with $A$ the (unit) cross-sectional area of the system. Multidimensional formulations in the spirit of (3) have been derived by Chang and Gao [1995], Mühlhaus and Oka [1996] and Suiker et al. [2001a; Suiker et al. [2001b], among others.

Apart from the lowest-order, standard terms, (3) also contains higher-order terms proportional to $d^{2}, d^{4}$, etc. These additional terms capture the microstructural effects that are present in the discrete model of (1) but that are absent in standard continuum theories as retrieved by taking $d=0$ in (3). The simplest continuum model that incorporates microstructural effects is obtained by truncating the series in (3) after the term that is proportional to $d^{2}$; unfortunately, such a model is unstable and its solutions in a boundary-value problem may lack uniqueness [Askes et al. 2002]. Although stability and uniqueness can be restored by incorporating the next term, i.e., truncating after the $d^{4}$ term, the numerical implementation of such a model is complicated [Askes et al. 2002]; thus, alternative solution strategies are explored here.

\section{Stabilising the continuum equations}

Unstable gradient theories can be turned into stable gradient theories by means of Padé approximations, as has been explored in [Andrianov et al. 2003; Andrianov and Awrejcewicz 2008; Andrianov et al. 2010]. However, there are various ways to do this, and the format of the resulting equations depends on which equations are transformed by the Padé approximation.

3.1. Irreducible form. Firstly, (3) is truncated after the first nonstandard term. The various spatial derivatives are factorised as

$$
\rho \ddot{u}=\left(1+\frac{1}{12} d^{2} \frac{\partial^{2}}{\partial x^{2}}\right) E \frac{\partial^{2} u}{\partial x^{2}} .
$$

A [0,1]-Padé approximation is used according to

$$
1+a \approx \frac{1}{1-a} \text { for } a \ll 1
$$

For $a$ in (5), we will substitute the operator $\frac{1}{12} d^{2} \partial^{2} / \partial x^{2}$, which allows us to rewrite (4) as

$$
\left(1-\frac{1}{12} d^{2} \frac{\partial^{2}}{\partial x^{2}}\right) \rho \ddot{u}=E \frac{\partial^{2} u}{\partial x^{2}} \text {. }
$$


The higher-order gradient term now appears on the inertia side of the equation, and for this reason, it has been called microinertia, internal inertia or higher-order inertia in the literature [Vardoulakis and Aifantis 1994; Wang and Sun 2002; Bennett et al. 2007]. Equation (6), or slight variations thereof, has also been obtained by various other researchers using asymptotic series equivalence; see for instance the work of Rubin et al. [1995], Chen and Fish [2001] and Pichugin et al. [2008].

Note that the only unknown appearing in (6) is the displacement; for this reason, this format is denoted as irreducible. Although at first sight it may appear that the micromechanical background of the higher-order terms is lost through the Padé approximation, an alternative interpretation of the microinertia contribution in terms of long-range interactions has been provided in [Askes and Gitman 2014].

3.2. Reducible form. It is also possible to extract a (one-dimensional) relation between stress $\sigma$ and strain $\varepsilon$ from (3) such that

$$
\rho \ddot{u}=\frac{\partial \sigma}{\partial x} \quad \text { and } \quad \varepsilon=\frac{\partial u}{\partial x} .
$$

The stress-strain relation then follows as

$$
\sigma=E\left(\varepsilon+\frac{1}{12} d^{2} \frac{\partial^{2} \varepsilon}{\partial x^{2}}\right)=E\left(1+\frac{1}{12} d^{2} \frac{\partial^{2}}{\partial x^{2}}\right) \varepsilon
$$

where series have again been truncated after the first nonstandard term. Applying the $[0,1]$-Padé approximation to (8) yields

$$
\left(1-\frac{1}{12} d^{2} \frac{\partial^{2}}{\partial x^{2}}\right) \sigma=E \varepsilon
$$

Equations (7) and (9) can be combined into a system of coupled equations,

together with

$$
\rho \ddot{u}=\frac{\partial \sigma}{\partial x}
$$

$$
\sigma-\frac{1}{12} d^{2} \frac{\partial^{2} \sigma}{\partial x^{2}}=E \frac{\partial u}{\partial x}
$$

where the unknowns are the displacement $u$ as well as the stress $\sigma$. In contrast to the single fourth-order equation (6), (10) is a set of two second-order equations. They are termed reducible because it is possible to eliminate one of the unknowns, namely the stress $\sigma$. To do this, the second-order spatial derivative of (10a) must be taken and, multiplied with $\frac{1}{12} d^{2}$, subtracted from the original expression (10a):

$$
\rho\left(\ddot{u}-\frac{1}{12} d^{2} \frac{\partial^{2} \ddot{u}}{\partial x^{2}}\right)=\frac{\partial}{\partial x}\left(\sigma-\frac{1}{12} d^{2} \frac{\partial^{2} \sigma}{\partial x^{2}}\right) .
$$

If $(10 b)$ is substituted into the right-hand side of (11), the stress will disappear 
from the expressions and thus it is possible to retrieve (6). This reduction of the number of unknowns, and its consequences, will be discussed in more depth below in Section 4.2.

\section{Energy functionals for the multidimensional case}

Above, the governing equations have been derived from simple mechanical and mathematical arguments in a one-dimensional context. Next, we will show how the analogous multidimensional equations can be derived from variational principles. Hamilton's action $S$ is defined as

$$
S=\int_{t_{0}}^{t_{1}} L \mathrm{~d} t
$$

The governing equations of the models can be derived by requiring stationarity of $S$, that is, $\delta S=0$. The energy functional (or Lagrangian function) $L$ is defined individually for the two different models below, but we will assume that $L$ depends on the displacements $u_{i}$ and their spatial and temporal derivatives, as well as on the stresses $\sigma_{i j}$ and their spatial derivatives:

$$
L=L\left(u_{i} ; u_{i, j} ; \dot{u}_{i} ; \dot{u}_{i, j} ; \sigma_{i j} ; \sigma_{i j, k}\right) .
$$

Substituting (13) into (12) and requiring $\delta S=0$ yields

$$
\begin{aligned}
\int_{t_{0}}^{t_{1}} & \delta L \mathrm{~d} t \\
& =\int_{t_{0}}^{t_{1}}\left(\delta u_{i} \frac{\partial L}{\partial u_{i}}+\delta u_{i, j} \frac{\partial L}{\partial u_{i, j}}+\delta \dot{u}_{i} \frac{\partial L}{\partial \dot{u}_{i}}+\delta \dot{u}_{i, j} \frac{\partial L}{\partial \dot{u}_{i, j}}+\delta \sigma_{i j} \frac{\partial L}{\partial \sigma_{i j}}+\delta \sigma_{i j, k} \frac{\partial L}{\partial \sigma_{i j, k}}\right) \mathrm{d} t \\
& =0,
\end{aligned}
$$

which, as usual, can be rewritten as

$$
\begin{aligned}
\int_{t_{0}}^{t_{1}} \delta u_{i} & \left(\frac{\partial L}{\partial u_{i}}-\frac{\partial}{\partial x_{j}} \frac{\partial L}{\partial u_{i, j}}-\frac{\partial}{\partial t} \frac{\partial L}{\partial \dot{u}_{i}}+\frac{\partial^{2}}{\partial x_{j} \partial t} \frac{\partial L}{\partial \dot{u}_{i, j}}\right) \mathrm{d} t \\
+\int_{t_{0}}^{t_{1}} & \frac{\partial}{\partial x_{j}}\left(\delta u_{i} \frac{\partial L}{\partial u_{i, j}}-\delta u_{i} \frac{\partial}{\partial t} \frac{\partial L}{\partial \dot{u}_{i, j}}\right) \mathrm{d} t+\int_{t_{0}}^{t_{1}} \frac{\partial}{\partial t}\left(\delta u_{i} \frac{\partial L}{\partial \dot{u}_{i}}+\delta u_{i, j} \frac{\partial L}{\partial \dot{u}_{i, j}}\right) \mathrm{d} t \\
& \quad+\int_{t_{0}}^{t_{1}} \delta \sigma_{i j}\left(\frac{\partial L}{\partial \sigma_{i j}}-\frac{\partial}{\partial x_{k}} \frac{\partial L}{\partial \sigma_{i j, k}}\right) \mathrm{d} t+\int_{t_{0}}^{t_{1}} \frac{\partial}{\partial x_{k}}\left(\delta \sigma_{i j} \frac{\partial L}{\partial \sigma_{i j, k}}\right) \mathrm{d} t=0
\end{aligned}
$$

The third integral cancels through the requirement that $\delta u_{i}=0$ and $\delta u_{i, j}=0$ for $t=t_{0}$ and for $t=t_{1}$. The first and fourth integrals will lead to field equations, whereas the second and fifth will contribute to the natural boundary conditions. 
4.1. Irreducible form. The Lagrangian function of the irreducible form can be written as

$$
\begin{aligned}
L_{\text {irred }}=\int_{\Omega} \frac{1}{2} \rho\left(\dot{u}_{i} \dot{u}_{i}+\ell^{2} \dot{u}_{i, j} \dot{u}_{i, j}\right) \mathrm{d} V-\int_{\Omega} \frac{1}{2} u_{i, j} C_{i j k l} u_{k, l} \mathrm{~d} V & \\
& +\int_{\Omega} u_{i} b_{i} \mathrm{~d} V+\int_{\Gamma_{n}} u_{i} t_{i} \mathrm{~d} S
\end{aligned}
$$

where the first integral is the kinetic energy, the second integral is the stored strain energy and the last two terms represent the work of the external forces. Thus, for this model, the Lagrangian takes the usual format of "kinetic energy minus potential energy", whereby the nonstandard contributions are included in the kinetic energy only [Lazar and Anastassiadis 2007; Polizzotto 2012]. Note that for the internal length scale we have now used the generic notation $\ell$ rather than the notation $d$ that was used in the previous section in relation to the discrete model.

Substituting (16) into (15) and noting that $\delta u_{i}=0$ on $\Gamma_{e}$ leads to

$$
\begin{aligned}
\int_{t_{0}}^{t_{1}} \int_{\Omega} \delta u_{i}\left(b_{i}+C_{i j k l} u_{k, j l}-\rho \ddot{u}_{i}+\rho \ell^{2} \ddot{u}_{i, j j}\right) \mathrm{d} V \mathrm{~d} t \\
+\int_{t_{0}}^{t_{1}} \int_{\Gamma_{n}} \delta u_{i}\left(t_{i}-n_{j}\left(C_{i j k l} u_{k, l}+\rho \ell^{2} \ddot{u}_{i, j}\right)\right) \mathrm{d} S \mathrm{~d} t=0,
\end{aligned}
$$

where, as usual, the boundary $\Gamma$ of the domain $\Omega$ is decomposed into parts $\Gamma_{n}$ and $\Gamma_{e}$ associated with natural and essential boundary conditions: $\Gamma=\Gamma_{n} \cup \Gamma_{e}$ and $\varnothing=\Gamma_{n} \cap \Gamma_{e}$.

A symmetric Hookean stress $\tau_{i j}^{\mathrm{H}}=C_{i j k l} u_{k, l}$ can be identified in terms of which the field equations and natural boundary conditions can be written as

$$
\begin{array}{ll}
\rho\left(\ddot{u}_{i}-\ell^{2} \ddot{u}_{i, j j}\right)=\tau_{i j, j}^{\mathrm{H}}+b_{i} & \text { in } \Omega, \\
n_{j}\left(\tau_{i j}^{\mathrm{H}}+\rho \ell^{2} \ddot{u}_{i, j}\right)=t_{i} & \text { on } \Gamma_{n} .
\end{array}
$$

In our opinion, Hookean stress is appropriate terminology for $\tau_{i j}^{\mathrm{H}}$, not Cauchy stress, since the equations of motion and the natural boundary conditions contain additional gradients of the acceleration that are not included in the definition of $\tau_{i j}^{\mathrm{H}}$. In Appendix A this particular terminology is motivated.

Remark. A nonsymmetric stress tensor $\tau_{i j}^{\mathrm{B}}$ can be identified as (see [Lazar and Anastassiadis 2007])

$$
\tau_{i j}^{\mathrm{B}}=C_{i j k l} u_{k, l}+\rho \ell^{2} \ddot{u}_{i, j}
$$

This would enable one to write the equations of motion and natural boundary conditions in terms of a stress tensor that is similar in role to a standard Cauchy stress as explained in Appendix A. However, since $\tau_{i j}^{\mathrm{B}}$ is nonsymmetric, using the term Cauchy stress for this tensor is not obvious. This issue of nomenclature is left for future debate and discussion. 
4.2. Reducible form. For the reducible form, the Lagrangian function adopts a less common appearance, which, to the authors' best knowledge, is novel:

$$
\begin{array}{r}
L_{\text {red }}=\int_{\Omega} \frac{1}{2} \rho \dot{u}_{i} \dot{u}_{i} \mathrm{~d} V-\int_{\Omega} u_{i, j} \sigma_{i j} \mathrm{~d} V+\int_{\Omega} \frac{1}{2}\left(\sigma_{i j} S_{i j k l} \sigma_{k l}+\ell^{2} \sigma_{i j, m} S_{i j k l} \sigma_{k l, m}\right) \mathrm{d} V \\
+\int_{\Omega} u_{i} b_{i} \mathrm{~d} V+\int_{\Gamma_{n}} u_{i} t_{i} \mathrm{~d} S,
\end{array}
$$

where $S_{i j k l}$ is the elastic compliance tensor. The first integral is again the kinetic energy, whilst the last two integrals contain the external work. The third integral contains the stored complementary energy with a positive rather than negative sign, but the effects of the lower-order part are offset by the effects of the second integral, which couples the effects of the two sets of unknowns, namely displacements and stresses. In the reducible form, the displacement derivative $u_{i, j}$ is no longer energyconjugated to the (symmetric) stress $\sigma_{i j}$, unless $\ell=0$. Therefore, the second integrand does not have the meaning of internal work. Expression (20) can also be rewritten as a Hellinger-Reissner functional whereby the displacements act as Lagrange multipliers to enforce balance of momentum in $\Omega$ and on $\Gamma$ [Askes and Gutiérrez 2006; Polizzotto 2015].

Again making use of $\delta u_{i}=0$ on $\Gamma_{e}$, substitution of (20) into (15) yields

$$
\begin{aligned}
\int_{t_{0}}^{t_{1}} \int_{\Omega} \delta u_{i}\left(b_{i}+\right. & \left.\sigma_{i j, j}-\rho \ddot{u}_{i}\right) \mathrm{d} V \mathrm{~d} t+\int_{t_{0}}^{t_{1}} \int_{\Gamma_{n}} \delta u_{i}\left(t_{i}-n_{j} \sigma_{i j}\right) \mathrm{d} S \mathrm{~d} t \\
& +\int_{t_{0}}^{t_{1}} \int_{\Omega} \delta \sigma_{i j}\left(-u_{i, j}+S_{i j k l} \sigma_{k l}-\ell^{2} S_{i j k l} \sigma_{k l, m m}\right) \mathrm{d} V \mathrm{~d} t \\
& \quad+\int_{t_{0}}^{t_{1}} \oint_{\Gamma} \delta \sigma_{i j} n_{m} S_{i j k l} \sigma_{k l, m} \mathrm{~d} S \mathrm{~d} t=0
\end{aligned}
$$

so that the following set of coupled governing equations can be identified:

$$
\begin{array}{ll}
\rho \ddot{u}_{i}=\sigma_{i j, j}+b_{i} & \text { in } \Omega, \\
n_{j} \sigma_{i j}=t_{i} & \text { on } \Gamma_{n}, \\
S_{i j k l}\left(\sigma_{k l}-\ell^{2} \sigma_{k l, m m}\right)=\frac{1}{2}\left(u_{i, j}+u_{j, i}\right) & \text { in } \Omega, \\
n_{m} \ell^{2} S_{i j k l} \sigma_{k l, m}=0 & \text { on } \Gamma .
\end{array}
$$

From the format of (22a) and (22b), it is clear that the meaning of $\sigma_{i j}$ in the reducible model is that of the Cauchy stress. Equations (22) have also been derived, using different arguments, by Eringen [1983]; see Appendix B for a discussion.

Equations (22) form a set of coupled equations with independent unknowns $u_{i}$ and $\sigma_{i j}$, but they are reducible in the sense that it is possible to eliminate the stresses $\sigma_{i j}$. To do so, firstly the Laplacian of (22a) is taken and multiplied with $\ell^{2}$, after which the result is subtracted from the original expression (22a). This gives

$$
\rho\left(\ddot{u}_{i}-\ell^{2} \ddot{u}_{i, j j}\right)=\sigma_{i j, j}-\ell^{2} \sigma_{i j, k k}+b_{i}-\ell^{2} b_{i, j j} .
$$


Next, (22c) is premultiplied with the elastic stiffness tensor $C_{i j k l}$ and substituted into (23), leading to

$$
\rho\left(\ddot{u}_{i}-\ell^{2} \ddot{u}_{i, j j}\right)=C_{i j k l} u_{k, j l}+b_{i}-\ell^{2} b_{i, j j},
$$

which is equivalent to (18a) except for the presence of the Laplacian of the body forces $b_{i, j}$ and a mismatch in the associated variationally consistent boundary conditions. Note that the effect of the higher-order gradients disappears altogether in statics in the case $b_{i, j j}=0$.

Remark. From (22c) it is clear that the gradient enrichment affects the constitutive part of the field equations, and therefore the term "gradient elasticity" seems appropriate for what is here denoted as the reducible form. In contrast, it could be argued that using the term "gradient elasticity" is less suitable for the irreducible format represented in (24), because the gradient enrichment operates on the accelerations, not stresses or strains - i.e., the elasticity part of the irreducible form retains its classical format. However, we still prefer to refer to the irreducible form as a particular variant of gradient elasticity, because of the close relation between the reducible form and the irreducible form. Due to the coupling between the equations of motion and the constitutive equations, the gradient enrichment of the accelerations will affect the stresses and strains, albeit indirectly.

\section{Finite element equations}

In order to obtain solutions of the relevant partial differential equations for domains of arbitrary geometry, a numerical solution strategy is required. Here, the finite element method will be used for the spatial discretisation, whereas the Newmark time integrator will be adopted to progress the solution in the time domain. The finite element equations of the irreducible form are well established and need not be revisited here - the interested reader is referred to [Fish et al. 2002a; 2002b; Askes and Aifantis 2011].

For the reducible form, we write $\underline{u}=\boldsymbol{N}_{u} \boldsymbol{d}$ and $\underline{\sigma}=\boldsymbol{N}_{\sigma} \boldsymbol{S}$ where $\underline{u}$ and $\underline{\sigma}$ are column vectors containing the relevant components of the displacements and Cauchy stresses, respectively. Furthermore, the matrices $N_{u}$ and $N_{\sigma}$ contain the shape functions for displacements and Cauchy stresses whereas $\boldsymbol{d}$ and $\boldsymbol{s}$ are the nodal displacements and nodal Cauchy stresses. The spatial discretisation of (20) can thus be written as

$$
\begin{aligned}
& L_{\mathrm{red}}^{\mathrm{FE}}=\int_{\Omega} \frac{1}{2} \rho \dot{\boldsymbol{d}}^{T} \boldsymbol{N}_{u}^{T} \boldsymbol{N}_{u} \dot{\boldsymbol{d}} \mathrm{d} V-\int_{\Omega} \boldsymbol{d}^{T} \boldsymbol{B}_{u}^{T} \boldsymbol{N}_{\sigma} \boldsymbol{s} \mathrm{d} V \\
& +\int_{\Omega} \frac{1}{2} \boldsymbol{s}^{T}\left(\boldsymbol{N}_{\sigma}^{T} \boldsymbol{S} \boldsymbol{N}_{\sigma}+\sum_{i=1}^{3} \ell^{2} \frac{\partial \boldsymbol{N}_{\sigma}^{T}}{\partial x_{i}} \boldsymbol{S} \frac{\partial \boldsymbol{N}_{\sigma}}{\partial x_{i}}\right) \boldsymbol{s} \mathrm{d} V+\int_{\Omega} \boldsymbol{d}^{T} \boldsymbol{N}_{u}^{T} \underline{b} \mathrm{~d} V+\int_{\Gamma_{n}} \boldsymbol{d}^{T} \boldsymbol{N}_{u}^{T} \underline{t} \mathrm{~d} S
\end{aligned}
$$


where $\underline{b}$ and $\underline{t}$ contain the components of the distributed body and surface forces, respectively. Furthermore, $\boldsymbol{B}_{u}$ is the standard strain-displacement matrix with derivatives of the displacement shape functions $\boldsymbol{N}_{u}$ and $S$ is the matrix counterpart of the compliance tensor $S_{i j k l}$.

Requiring $\delta L_{\text {red }}^{\mathrm{FE}}=0$ leads to a system of finite element equations according to

$$
\left[\begin{array}{cc}
\boldsymbol{M}_{u и} & \mathbf{0} \\
\mathbf{0} & \mathbf{0}
\end{array}\right]\left[\begin{array}{c}
\ddot{\boldsymbol{d}} \\
\ddot{\boldsymbol{s}}
\end{array}\right]+\left[\begin{array}{cc}
\mathbf{0} & \boldsymbol{K}_{u \sigma} \\
\boldsymbol{K}_{\sigma u} & \boldsymbol{K}_{\sigma \sigma}
\end{array}\right]\left[\begin{array}{l}
\boldsymbol{d} \\
\boldsymbol{s}
\end{array}\right]=\left[\begin{array}{l}
\boldsymbol{f} \\
\mathbf{0}
\end{array}\right]
$$

where

$$
\begin{aligned}
\boldsymbol{M}_{u u} & =\int_{\Omega} \rho \boldsymbol{N}_{u}^{T} \boldsymbol{N}_{u} \mathrm{~d} V, \\
\boldsymbol{K}_{u \sigma}=\boldsymbol{K}_{\sigma u}^{T} & =\int_{\Omega} \boldsymbol{B}_{u}^{T} \boldsymbol{N}_{\sigma} \mathrm{d} V, \\
\boldsymbol{K}_{\sigma \sigma} & =-\int_{\Omega}\left(\boldsymbol{N}_{\sigma}^{T} \boldsymbol{S} \boldsymbol{N}_{\sigma}+\sum_{i=1}^{3} \ell^{2} \frac{\partial \boldsymbol{N}_{\sigma}^{T}}{\partial x_{i}} \boldsymbol{S} \frac{\partial \boldsymbol{N}_{\sigma}}{\partial x_{i}}\right) \mathrm{d} V .
\end{aligned}
$$

Finite-element implementation of (26) was carried out using the recommendations of the statics theory given in [Askes and Gutiérrez 2006], in particular the use of quadratic shape functions for $\boldsymbol{s}$ and linear shape functions for $\boldsymbol{d}$. This particular choice of shape functions avoids oscillations in the displacement field, although a formal investigation of the inf-sup condition may require further refinement of the two sets of interpolations.

\section{Numerical example}

Although the reducible form can be transformed into the irreducible form as shown in (23) and (24), the associated change in variationally consistent boundary conditions has implications when it comes to the simulation of crack tip stresses. This will be demonstrated by means of the numerical example shown in Figure 2.

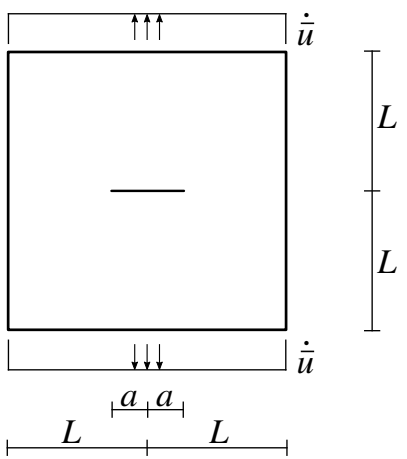

Figure 2. Strip with central crack: geometry and loading conditions. 
A square strip with dimension $2 L=2 \mathrm{~m}$ has a central crack of length $2 a=0.5 \mathrm{~m}$. The material properties are mass density $\rho=1 \mathrm{~kg} / \mathrm{m}^{3}$, Young's modulus $E=$ $100 \mathrm{~N} / \mathrm{m}^{2}$ and Poisson's ratio $v=\frac{1}{4}$, whilst a plane stress assumption has been made. Furthermore, the gradient elasticity length scale $\ell=0.1 \mathrm{~m}$. The strip is subjected to outward vertical velocities $\dot{\bar{u}}=10 \mathrm{~m} / \mathrm{s}$ imposed on the top and bottom edges, as indicated, which leads to stress waves propagating towards the centre of the strip. Away from the crack, the stress waves will have the shape of a block wave due to the nature of the loading conditions, but the presence of the crack will disturb this pattern, and indeed in a classical elasticity setting, this will lead to singular stresses and strains at the tips of the crack. It is the aim of this example to verify whether these singularities can be avoided in the reducible and irreducible formulations of gradient elasticity discussed above. For reasons of symmetry, only the top quarter of the strip is modelled.

The irreducible format of gradient elasticity is implemented with four-noded quadrilateral elements for the displacements. The reducible format is implemented with eight-noded elements for the stresses and four-noded quadrilateral elements for the displacements - see [Askes and Gutiérrez 2006] for details on this particular choice. Structured finite element meshes consisting of square elements are used, and a sequence of uniformly refined meshes is taken to monitor the behaviour of the stresses at the crack tip. Since in the irreducible format the stresses are postprocessed from linear displacements whereas in the reducible format the stresses are primary unknowns interpolated with quadratic shape functions, there is an obvious mismatch in stress resolution between the two formats. To address this mismatch, the meshes used range from $16 \times 16$ to $128 \times 128$ elements for the irreducible format, whereas they range from $8 \times 8$ to $64 \times 64$ for the reducible format.

Regarding the imposition of traction boundary conditions, it must be realised that the stresses are primary variables in the reducible formulation, whereas they are derived quantities in the irreducible formulation. In the reducible formulation, traction boundary conditions are thus essential boundary conditions and are imposed by assigning prescribed values to the relevant stress components (e.g., $\sigma_{y y}=0$ on the crack face). On the other hand, traction boundary conditions are natural boundary conditions in the irreducible formulation; applying zero tractions on the crack face means that the left-hand-side of (18b) is set equal to zero, which is handled straightforwardly in a finite element context. Finally, and for the sake of completeness, it is noted that displacement (and velocity) boundary conditions have been implemented using Lagrange Multipliers in the reducible formulation.

The Newmark constant average acceleration scheme is used for the time integration. This scheme is unconditionally stable; therefore, the only criterion for selecting the time step is accuracy. Following the recommendations given in [Askes et al. 2008; Bennett and Askes 2009], the time step is chosen such that waves 


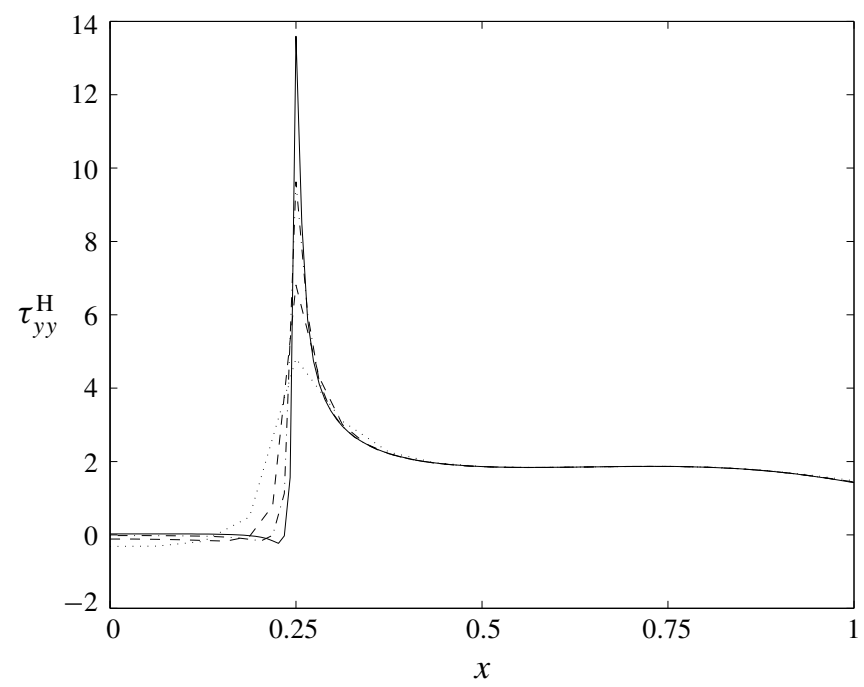

Figure 3. Vertical normal stress $\tau_{y y}^{\mathrm{H}}\left(\mathrm{N} / \mathrm{m}^{2}\right)$ versus $x(\mathrm{~m})$ for the irreducible format $-16 \times 16$ elements (dotted), $32 \times 32$ elements (dashed), $64 \times 64$ elements (dot-dashed) and $128 \times 128$ elements (solid).

propagate approximately half an element per time step. Time domain simulations were carried out from time $t=0 \mathrm{~s}$ to $t=0.2 \mathrm{~s}$.

Figures 3 and 4 show the profiles of the vertical normal stress for both formats and the indicated range of finite element meshes, where the origin of the coordinate system is chosen at the centre of the crack. For the irreducible format (Figure 3), we have plotted the Hookean stress $\tau_{y y}^{\mathrm{H}}$ (see Section 4.1) whilst for the reducible format the Cauchy stress $\sigma_{y y}$ is plotted (Figure 4).

The stress profiles for the irreducible formulation appear to converge towards a unique solution, except for the crack tip value. At the crack tip, the stress increases significantly for every refinement of the mesh. This is an indication that a stress singularity is present at the crack tip. To analyse this in more depth, Richardson extrapolations have been carried out for the crack tip stresses. Table 1 reports the

\begin{tabular}{|crc|}
\hline mesh & $\tau_{y y}^{\mathrm{H}}$ & extrapolation \\
\hline $16 \times 16$ & 4.8077 & \\
$32 \times 32$ & 6.8091 & 8.8105 \\
$64 \times 64$ & 9.6223 & 13.6438 \\
$128 \times 128$ & 13.5923 & 20.0751 \\
\hline
\end{tabular}

Table 1. Crack tip stress and Richardson extrapolation in $\mathrm{N} / \mathrm{m}^{2}$ for irreducible form. 


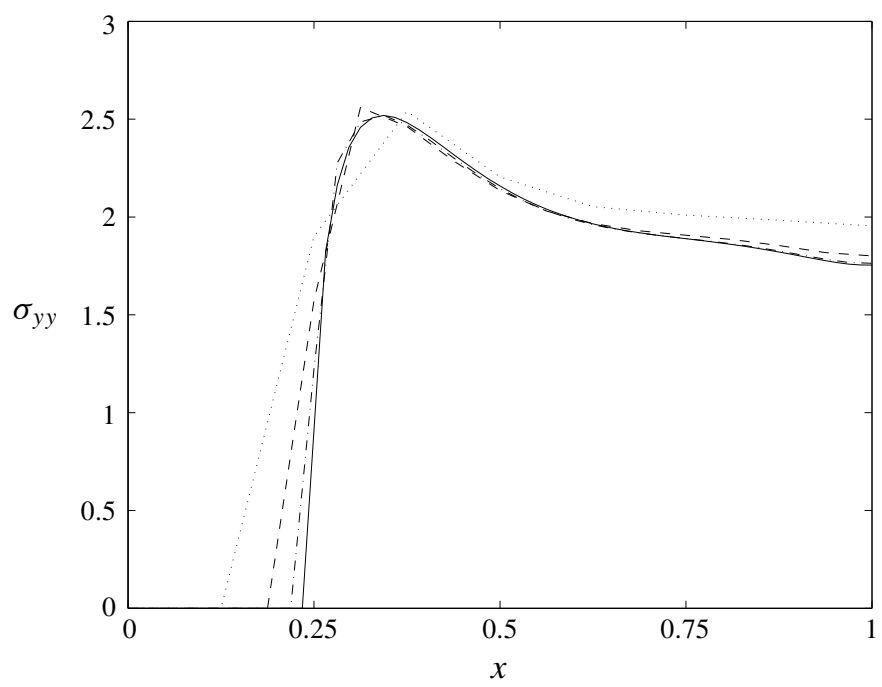

Figure 4. Vertical normal stress $\sigma_{y y}\left(\mathrm{~N} / \mathrm{m}^{2}\right)$ versus $x(\mathrm{~m})$ for the reducible format $-8 \times 8$ elements (dotted), $16 \times 16$ elements (dashed), $32 \times 32$ elements (dot-dashed) and $64 \times 64$ elements (solid).

values of the crack tip stress and their extrapolations. (The first extrapolation is a two-point extrapolation based on the coarsest two meshes, the second is a threepoint extrapolation based on the coarsest three meshes, and mutatis mutandis for the last extrapolation.) The numerical results confirm that the crack tip stress grows in a seemingly unbounded manner, whereas the difference between numerical stress and extrapolated stress increases with refinement of the mesh. This confirms the suggestion that a singularity is present. Thus, it must be concluded that the irreducible format is not capable of avoiding stress singularities. This is reported for the Hookean stress $\tau_{y y}^{\mathrm{H}}$ but will carry over to the pseudo Cauchy stress $\tau_{y y}^{\mathrm{B}}$ since the latter quantity includes the former.

On the other hand, the results of the reducible format clearly converge towards a unique, nonsingular solution, and the singularities that plague classical elasticity formulations are avoided. However, it must be noted that the maximum stress occurs not at the crack tip but further inside the material. This is in line with the analysis and results reported in [Simone et al. 2004].

\section{Conclusions}

We have reviewed and systematically compared two formats of gradient elasticity. Both formats can be derived by continualising a one-dimensional discrete model and stabilising the resulting equations, but the models differ in respect of which particular equation is stabilised - either the field equation (leading to what 
is denoted as the "irreducible format") or the constitutive equation (leading to the "reducible format"). The multidimensional case, including the associated boundary conditions, has been derived from a variational principle. It is noted that the field equations of the irreducible format can be retrieved from those of the reducible format (assuming that the Laplacian of the body forces vanishes), but the variationally consistent boundary conditions are different for the two models.

This has implications for the solution of initial-boundary-value problems. We have presented a crack problem, and it was demonstrated that the irreducible format is not capable of avoiding singularities in the stress field. On the other hand, no singularities were found when the reducible format was used. Thus, for the dynamic analysis of stresses around sharp cracks, the reducible format is to be preferred.

\section{Appendix A: Nomenclature in gradient elasticity: Cauchy stress}

In the literature, there is a lack of consistency in which quantity is denoted as the Cauchy stress in gradient elasticity theories. Some eminent authors have used this term to indicate the derivative of the strain energy density with respect to the strain - see for instance [Mindlin 1964, p. 57] or [Shu et al. 1999, p. 375]. However, we have followed the arguments set out by Borino and Polizzotto [2003, Remark 3], who state that the term Cauchy stress should be used for the total stress quantity as it appears in the equilibrium equations; conversely, we have used the term Hookean stress for the derivative of the strain energy density with respect to the strain. We believe the former is in line with the conceptualisation of Cauchy himself, who discussed stresses as forming equilibrium (or indeed accelerating) systems by acting on surfaces, rather than as derivatives of energy functionals see for instance [Cauchy $1823 ; 1827 ; 1843$ ].

However, it is also noted that extending the concept of Cauchy stress as "force divided by area" to gradient-enriched continua leads, in general, to much more complicated expressions. This is illustrated by the format of the natural boundary conditions in Mindlin's [1964, pp. 67-68] theory of gradient elasticity. Askes and Metrikine [2005] as well as Froiio et al. [2010] have provided physical interpretations of the nonstandard boundary conditions.

\section{Appendix B: Eringen's 1983 differential theory of nonlocal elasticity}

The reducible format presented in Section 4 has been derived earlier in [Eringen 1983] from an integral formulation. Because the coupled nature of the governing equations of Eringen's theory is not always appreciated, it is worthwhile to summarise Eringen's theory. Adopting his notation unless stated otherwise, the equations of motion are given by [Eringen 1983, (2.1)] as

$$
t_{k l, k}+\rho\left(f_{l}-\ddot{u}_{l}\right)=0
$$


where $t_{k l}$ is the Cauchy stress tensor and $f_{l}$ is the body force density. With the restriction to isotropic linear elasticity, a Hookean stress $\sigma_{k l}^{0}$ is defined via [Eringen 1983, (2.3) and (2.4)] as

$$
\sigma_{k l}^{0}=\lambda \delta_{k l} u_{j, j}+\mu u_{k, l}+\mu u_{l, k}
$$

where a superscript 0 is included in $\sigma^{0}$ to avoid confusion with the Cauchy stress of the reducible theory discussed in Section 4.2. Furthermore, $\lambda$ and $\mu$ are the Lamé constants and $\delta_{k l}$ is the Kronecker delta.

The field equations are completed by a differential relation between the Cauchy stress $t_{k l}$ and the Hookean stress $\sigma_{k l}^{0}$. The particular relation that seems to have attracted most interest in the literature is given in [Eringen 1983, (3.19)] as

$$
t_{k l}-\ell^{2} t_{k l, j j}=\sigma_{k l}^{0}
$$

where the higher-order coefficient is simply indicated by $\ell^{2}$ (Eringen uses a more intricate notation with multiple symbols, which are not required in the present discussion).

Eringen [1983, pp. 4704-4705] also discusses the elimination of the stress $t_{k l}$ from the system of equations. Combining (3.13) and (3.18), he arrives at the irreducible form

$$
\sigma_{k l, k}^{0}+\left(1-\ell^{2} \nabla^{2}\right)\left(\rho f_{l}-\rho \ddot{u}_{l}\right)=0 .
$$

Next, he notes that the particular case of statics with vanishing body forces leads to

$$
\sigma_{k l, k}^{0}=0 .
$$

However, regarding natural boundary conditions, Eringen [1983, p. 4704] explicitly states that "[b]oundary conditions involving tractions [are] based on the stress tensor $t_{k l}$, not on $\sigma_{k l}^{0}$ ", while Eringen [2002, p. 100] also emphasises that "the real stress is not $\sigma_{k l}^{0}$ but $t_{k l}$ " - in both quotations we have added the superscript 0 to $\sigma$ as explained above. This means that (32) cannot be used in isolation to solve general boundary-value problems involving prescribed tractions.

In summary, in our opinion, a divergence-free Hookean stress $\sigma^{0}$ should not be considered as a fundamental equation of the Eringen theory because, firstly, it can only be retrieved by making the assumptions of zero body force and zero acceleration and, secondly, it cannot be used to solve general equilibrium problems due to a lack of associated traction boundary conditions. In this respect, we disagree with Lazar and Polyzos [2015], who suggest that (32) is an equilibrium equation in its own right - although these authors do confirm that the correct natural boundary conditions are in terms of $t_{k l}$ rather than $\sigma_{k l}^{0}$. 


\section{References}

[Andrianov 2002] I. V. Andrianov, "The specific features of the limiting transition from a discrete elastic medium to a continuous one", J. Appl. Math. Mech. 66:2 (2002), 261-265.

[Andrianov and Awrejcewicz 2008] I. V. Andrianov and J. Awrejcewicz, "Continuous models for 2D discrete media valid for higher-frequency domain”, Comput. Struct. 86:1-2 (2008), 140-144.

[Andrianov et al. 2003] I. V. Andrianov, J. Awrejcewicz, and R. G. Barantsev, "Asymptotic approaches in mechanics: New parameters and procedures", Appl. Mech. Rev. 56:1 (2003), 87-110.

[Andrianov et al. 2010] I. V. Andrianov, J. Awrejcewicz, and D. Weichert, "Improved continuous models for discrete media”, Math. Probl. Eng. 2010 (2010), 986242.

[Askes and Aifantis 2011] H. Askes and E. C. Aifantis, "Gradient elasticity in statics and dynamics: an overview of formulations, length scale identification procedures, finite element implementations and new results", Int. J. Solids Struct. 48:13 (2011), 1962-1990.

[Askes and Gitman 2014] H. Askes and I. M. Gitman, "A computational mechanics perspective on long-range interactions in gradient elasticity with microinertia”, J. Mech. Behav. Mater. 23:1-2 (2014), 37-40.

[Askes and Gutiérrez 2006] H. Askes and M. A. Gutiérrez, "Implicit gradient elasticity", Internat. J. Numer. Methods Engrg. 67:3 (2006), 400-416.

[Askes and Metrikine 2005] H. Askes and A. V. Metrikine, "Higher-order continua derived from discrete media: continualisation aspects and boundary conditions", Int. J. Solids Struct. 42:1 (2005), 187-202.

[Askes et al. 2002] H. Askes, A. S. J. Suiker, and L. J. Sluys, "A classification of higher-order strain-gradient models: linear analysis", Arch. Appl. Mech. 72:2 (2002), 171-188.

[Askes et al. 2008] H. Askes, B. Wang, and T. Bennett, "Element size and time step selection procedures for the numerical analysis of elasticity with higher-order inertia", J. Sound Vibr. 314:3-5 (2008), 650-656.

[Bennett and Askes 2009] T. Bennett and H. Askes, "Finite element modelling of wave dispersion with dynamically consistent gradient elasticity", Comput. Mech. 43:6 (2009), 815-825.

[Bennett et al. 2007] T. Bennett, I. M. Gitman, and H. Askes, "Elasticity theories with higher-order gradients of inertia and stiffness for the modelling of wave dispersion in laminates", Int. J. Fract. 148:2 (2007), 185-193.

[Borino and Polizzotto 2003] G. Borino and C. Polizzotto, "Letter to the editor: 'Higher-order strain/higher-order stress gradient models derived from a discrete microstructure, with application to fracture', by C. S. Chang, H. Askes and L. J. Sluys; Engineering Fracture Mechanics 69 (2002), 1907-1924”, Eng. Fract. Mech. 70:9 (2003), 1219-1221.

[Cauchy 1823] A.-L. Cauchy, "Recherches sur l'équilibre et le mouvement intérieur des corps solides ou fluides, élastiques ou non élastiques", B. Soc. Philomat. 1823 (1823), 9-13.

[Cauchy 1827] A.-L. Cauchy, "Sur les relations qui existent dans l'état d'équilibre d'un corps solide ou fluide, entre les pressions ou tensions et les forces accélératrices", pp. 108-111 in Exercices de mathématiques, vol. 2, Bure Frères, Paris, 1827.

[Cauchy 1843] A.-L. Cauchy, "Note sur les pressions supportées, dans un corps solide ou fluide, par deux portions de surface très voisines, l'une extérieure, l'autre intérieure à ce même corps", $C$. $R$. Hebd. Acad. Sci. 16 (1843), 151-155.

[Chang and Gao 1995] C. S. Chang and J. Gao, "Second-gradient constitutive theory for granular material with random packing structure”, Int. J. Solids Struct. 32:16 (1995), 2279-2293. 
[Charlotte and Truskinovsky 2008] M. Charlotte and L. Truskinovsky, "Towards multi-scale continuum elasticity theory”, Contin. Mech. Thermodyn. 20 (2008), 133-161.

[Chen and Fish 2001] W. Chen and J. Fish, "A dispersive model for wave propagation in periodic heterogeneous media based on homogenization with multiple spatial and temporal scales", J. Appl. Mech. 68:2 (2001), 153-161.

[Eringen 1983] A. C. Eringen, "On differential equations of nonlocal elasticity and solutions of screw dislocation and surface waves”, J. Appl. Phys. 54:9 (1983), 4703-4710.

[Eringen 2002] A. C. Eringen, Nonlocal continuum field theories, Springer, New York, 2002.

[Fish et al. 2002a] J. Fish, W. Chen, and G. Nagai, "Non-local dispersive model for wave propagation in heterogeneous media: multi-dimensional case", Internat. J. Numer. Methods Engrg. 54:3 (2002), 347-363.

[Fish et al. 2002b] J. Fish, W. Chen, and G. Nagai, "Non-local dispersive model for wave propagation in heterogeneous media: one-dimensional case", Internat. J. Numer. Methods Engrg. 54:3 (2002), 331-346.

[Froiio et al. 2010] F. Froiio, A. Zervos, and I. Vardoulakis, "On natural boundary conditions in linear 2nd-grade elasticity", pp. 211-221 in Mechanics of generalized continua, edited by G. A. Maugin and A. V. Metrikine, Adv. Mech. Math. 21, Springer, New York, 2010.

[Ioannidou et al. 2001] T. Ioannidou, J. Pouget, and E. C. Aifantis, "Kink dynamics in a lattice model with long-range interactions", J. Phys. A 34:20 (2001), 4269-4280.

[Kwong and Gitman 2012] M. T. Kwong and I. M. Gitman, "Gradient elastic stress analysis for anisotropic bimaterial interface with arbitrarily oriented crack", Int. J. Fract. 173:1 (2012), 79-85.

[Lazar and Anastassiadis 2007] M. Lazar and C. Anastassiadis, "Lie point symmetries, conservation and balance laws in linear gradient elastodynamics", J. Elasticity 88:1 (2007), 5-25.

[Lazar and Polyzos 2015] M. Lazar and D. Polyzos, "On non-singular crack fields in Helmholtz type enriched elasticity theories", Int. J. Solids Struct. 62 (2015), 1-7.

[Mindlin 1964] R. D. Mindlin, "Micro-structure in linear elasticity", Arch. Rational Mech. Anal. 16 (1964), 51-78.

[Mühlhaus and Oka 1996] H. B. Mühlhaus and F. Oka, "Dispersion and wave propagation in discrete and continuous models for granular materials”, Int. J. Solids Struct. 33:19 (1996), 2841-2858.

[Pichugin et al. 2008] A. V. Pichugin, H. Askes, and A. Tyas, "Asymptotic equivalence of homogenisation procedures and fine-tuning of continuum theories", J. Sound Vibr. 313:3-5 (2008), 858-874.

[Polizzotto 2012] C. Polizzotto, "A gradient elasticity theory for second-grade materials and higher order inertia”, Int. J. Solids Struct. 49:15-16 (2012), 2121-2137.

[Polizzotto 2015] C. Polizzotto, "A unifying variational framework for stress gradient and strain gradient elasticity theories”, Eur. J. Mech. A Solids 49 (2015), 430-440.

[Rosenau 1984] P. Rosenau, "Dynamics of nonlinear mass-spring chains near the continuum limit", Phys. Lett. A 118:5 (1984), 222-227.

[Rubin et al. 1995] M. B. Rubin, P. Rosenau, and O. Gottlieb, "Continuum model of dispersion caused by an inherent material characteristic length”, J. Appl. Phys. 77:8 (1995), 4054-4063.

[Shu et al. 1999] J. Y. Shu, W. E. King, and N. A. Fleck, "Finite elements for materials with strain gradient effects", Internat. J. Numer. Methods Engrg. 44:3 (1999), 373-391.

[Simone et al. 2004] A. Simone, H. Askes, and L. J. Sluys, "Incorrect initiation and propagation of failure in non-local and gradient-enhanced media", Int. J. Solids Struct. 41:2 (2004), 351-363. 
[Suiker et al. 2001a] A. S. J. Suiker, R. de Borst, and C. S. Chang, "Micro-mechanical modelling of granular material, I: Derivation of a second-gradient micro-polar constitutive theory", Acta Mech. 149:1 (2001), 161-180.

[Suiker et al. 2001b] A. S. J. Suiker, A. V. Metrikine, and R. de Borst, "Comparison of wave propagation characteristics of the Cosserat continuum and corresponding discrete lattice models", Int. J. Solids Struct. 38:9 (2001), 1563-1583.

[Vardoulakis and Aifantis 1994] I. Vardoulakis and E. C. Aifantis, "On the role of microstructure in the behavior of soils: effects of higher order gradients and internal inertia", Mech. Mater. 18:2 (1994), 151-158.

[Wang and Sun 2002] Z.-P. Wang and C. T. Sun, "Modeling micro-inertia in heterogeneous materials under dynamic loading”, Wave Motion 36:4 (2002), 473-485.

Received 9 Mar 2016. Revised 22 Jul 2016. Accepted 26 Sep 2016.

HARM ASKES: h.askes@sheffield.ac.uk

Department of Civil and Structural Engineering, University of Sheffield, Sheffield, S1 3JD,

United Kingdom

INNA M. GITMAN: i.gitman@sheffield.ac.uk

Department of Mechanical Engineering, University of Sheffield, Sheffield, S1 3JD, United Kingdom

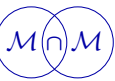


EDITORIAL BOARD

ANTONIO CARCATERRA

ERIC A. CARLEN

FRANCESCO DELL'ISOLA

RAFFAELE ESPOSITO

ALBERT FANNJIANG

Gilles A. FranCFORT

Pierangelo MARCATI

JEAN-JACQUES MARIGO

PETER A. MARKOWICH

MARTIN OSTOJA-STARZEWSKI

PIERRE SEPPECHER

DAVID J. STEIGMANN

PAUl STEINMANN

PierRe M. SuQueT

MANAGING EDITORS

MICOL AMAR

CORRADO LATTANZIO

ANGELA MADEO

MARTIN OSTOJA-STARZEWSKI

\section{ADVISORY BOARD}

ADNAN AKAY

Holm AltenBaCH

MICOL AMAR

HARM ASKES

TEODOR ATANACKOVIĆ

VICTOR BERDICHEVSKY

GUY BOUCHITTÉ

ANDREA BRAIDES

ROBERTO CAMASSA

MAURO CARFORE

ERIC DARVE

FELIX DARVE

ANNA DE MASI

GianPiEtro DEL Piero

EMMANUELE Di BENEDETTO

BERNOLD FIEDLER

IRENE M. GAMBA

DAVID Y. GAO

SERGEY GAVRILYUK

TIMOTHY J. HEALEY

DOMINIQUE JEULIN

ROGER E. KHAYAT

CORRADO LATTANZIO

ROBERT P. LIPTON

ANGELO LUONGO

ANGELA MADEO

JUAN J. MANFREDI

CARLO MARCHIORO

GÉRARD A. MAUGIN

ROBERTO NATALINI PATRIZIO NEFF

ANDREY PIATNITSKI

ERRICO PRESUTTI

MARIO PULVIRENTI

LUCIO RUSSO

Miguel A. F. SANJUAN

PATRICK SElvadurai

ALEXANDER P. SEYRANIAN

MIROSLAV ŠILHAVÝ

GUIDO SWEERS

ANTOINETTE TORDESILLAS

LEV TRUSKINOVSKY

JUAN J. L. VELÁZQUEZ VINCENZO VESPRI ANGELO VULPIANI msp.org/memocs

Università di Roma “La Sapienza”, Italia

Rutgers University, USA

(CO-CHAIR) Università di Roma "La Sapienza", Italia

(TREASURER) Università dell'Aquila, Italia

University of California at Davis, USA

(CO-CHAIR) Université Paris-Nord, France

Università dell'Aquila, Italy

École Polytechnique, France

DAMTP Cambridge, UK, and University of Vienna, Austria

(CHAIR MANAGING EDITOR) Univ. of Illinois at Urbana-Champaign, USA

Université du Sud Toulon-Var, France

University of California at Berkeley, USA

Universität Erlangen-Nürnberg, Germany

LMA CNRS Marseille, France

Università di Roma "La Sapienza", Italia

Università dell'Aquila, Italy

Université de Lyon-INSA (Institut National des Sciences Appliquées), France

(CHAIR MANAGING EDITOR) Univ. of Illinois at Urbana-Champaign, USA

Carnegie Mellon University, USA, and Bilkent University, Turkey

Otto-von-Guericke-Universität Magdeburg, Germany

Università di Roma "La Sapienza", Italia

University of Sheffield, UK

University of Novi Sad, Serbia

Wayne State University, USA

Université du Sud Toulon-Var, France

Università di Roma Tor Vergata, Italia

University of North Carolina at Chapel Hill, USA

Università di Pavia, Italia

Stanford University, USA

Institut Polytechnique de Grenoble, France

Università dell'Aquila, Italia

Università di Ferrara and International Research Center MEMOCS, Italia

Vanderbilt University, USA

Freie Universität Berlin, Germany

University of Texas at Austin, USA

Federation University and Australian National University, Australia

Université Aix-Marseille, France

Cornell University, USA

École des Mines, France

University of Western Ontario, Canada

Università dell' Aquila, Italy

Louisiana State University, USA

Università dell'Aquila, Italia

Université de Lyon-INSA (Institut National des Sciences Appliquées), France University of Pittsburgh, USA

Università di Roma "La Sapienza”, Italia

Université Paris VI, France

Istituto per le Applicazioni del Calcolo "M. Picone", Italy

Universität Duisburg-Essen, Germany

Narvik University College, Norway, Russia

Università di Roma Tor Vergata, Italy

Università di Roma "La Sapienza”, Italia

Università di Roma “Tor Vergata”, Italia

Universidad Rey Juan Carlos, Madrid, Spain

McGill University, Canada

Moscow State Lomonosov University, Russia

Academy of Sciences of the Czech Republic

Universität zu Köln, Germany

University of Melbourne, Australia

École Polytechnique, France

Bonn University, Germany

Università di Firenze, Italia

Università di Roma La Sapienza, Italia

MEMOCS (ISSN 2325-3444 electronic, 2326-7186 printed) is a journal of the International Research Center for the Mathematics and Mechanics of Complex Systems at the Università dell'Aquila, Italy.

Cover image: "Tangle” by $\odot$ John Horigan; produced using the Context Free program (contextfreeart.org).

PUBLISHED BY

7 mathematical sciences publishers

nonprofit scientific publishing

http://msp.org/

(C) 2017 Mathematical Sciences Publishers 
Mathematics and Mechanics of Complex Systems vol. 5 no. 1

Reducible and irreducible forms of stabilised gradient

elasticity in dynamics

Harm Askes and Inna M. Gitman

Dating Hypatia's birth: a probabilistic model

Canio Benedetto, Stefano Isola and Lucio Russo

On the possible effective elasticity tensors of 2-dimensional 41 and 3-dimensional printed materials

Graeme W. Milton, Marc Briane and Davit Harutyunyan Towards a complete characterization of the effective elasticity tensors of mixtures of an elastic phase and an almost rigid phase

Graeme W. Milton, Davit Harutyunyan and Marc Briane

MEMOCS is a journal of the International Research Center for the Mathematics and Mechanics of Complex Systems at the Università dell' Aquila, Italy.

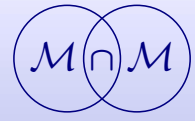

